\title{
When can Perfect State Transfer Occur?
}

\author{
Chris Godsil \\ Combinatorics \& Optimization \\ University of Waterloo \\ cgodsil@uwaterloo.ca
}

January 11, 2018

\begin{abstract}
Let $X$ be a graph on $n$ vertices with with adjacency matrix $A$ and let $H(t)$ denote the matrix-valued function $\exp (i A t)$. If $u$ and $v$ are distinct vertices in $X$, we say perfect state transfer from $u$ to $v$ occurs if there is a time $\tau$ such that $\left|H(\tau)_{u, v}\right|=1$. Our chief problem is to characterize the cases where perfect state transfer occurs. We show that if perfect state transfer does occur in a graph, then the square of its spectral radius is either an integer or lies in a quadratic extension of the rationals. From this we deduce that for any integer $k$ there only finitely many graphs with maximum valency $k$ on which perfect state transfer occurs. We also show that if perfect state transfer from $u$ to $v$ occurs, then the graphs $X \backslash u$ and $X \backslash v$ are cospectral and any automorphism of $X$ that fixes $u$ must fix $v$ (and conversely).
\end{abstract}

\section{Introduction}

Let $X$ be a graph on $n$ vertices with with adjacency matrix $A$ and let $H(t)$ denote the matrix-valued function $\exp (i A t)$. If $u$ and $v$ are distinct vertices in $X$, we say perfect state transfer from $u$ to $v$ occurs if there is a time $\tau$ such that $\left|H(\tau)_{u, v}\right|=1$. The terminology, and much of the motivation, comes from Quantum Physics, see e.g., [1]. We say that $X$ is periodic relative to a vertex $u$ if there is a time $\tau$ such that $\left|H(\tau)_{u, u}\right|=1$, and say $X$ itself is periodic if there is a time $\tau$ such that $\left|H(\tau)_{u, u}\right|=1$ for all vertices $u$. It is 
not hard to see that $X$ will be periodic with period $2 \pi$ if all its eigenvalues are integers. In [2] we showed that $X$ is periodic if and only if either its eigenvalues are integers, or $X$ is bipartite and there is a square-free integer $\Delta$ such that all eigenvalues of $X$ are integer multiples of $\sqrt{\Delta}$. As shown by Christandl et al [1] the $d$-cube is periodic, as are the Cartesian powers of the path $P_{3}$. (The eigenvalues of the $d$-cube are integers, and the eigenvalues of a Cartesian power of $P_{3}$ are integer multiples of $\sqrt{2}$.)

Our work in this paper has arisen from our attempts to characterize the pairs of vertices for which perfect state transfer does occur. Our results taken together imply that finding examples of perfect state transfer is not going to be easy. In particular we prove that if perfect state transfer from $u$ to $v$ occurs then

(1) Any automorphism of $X$ that fixes $u$ must fix $v$, and conversely.

(2) The subgraphs $X \backslash u$ and $X \backslash v$ are cospectral.

(3) If $\rho$ is the spectral radius of $X$, then $\rho^{2}$ is an integer.

\section{Periodicity and Perfect State Transfer}

Our first result is elementary, but it shows that perfect state transfer implies periodicity at at least two vertices. This is important conceptually, and also plays an important role in the rest of this paper. If $S$ is a subset of the vertices of the graph $X$ then we use $e_{S}$ to denote the characteristic vector of $S$. Here the only case of interest to us will be when $S$ is a single vertex, and then if $u \in V(X)$ then $e_{u}$ is one of the standard basis vectors.

2.1 Lemma. If perfect state transfer from $u$ to $v$ takes place at time $\tau$, then it also takes place from $v$ to $u$. Further $X$ is periodic with period $2 \tau$ at both $u$ and $v$.

Proof. We may assume that

$$
H(\tau) e_{u}=\gamma e_{v}
$$

where $|\gamma|=1$. Since $H(t)$ is symmetric for all $t$, we also have

$$
H(\tau) e_{v}=\gamma e_{u}
$$


and therefore

$$
H(2 \tau) e_{u}=\gamma^{2} e_{u}, \quad H(2 \tau) e_{v}=\gamma^{2} e_{v} .
$$

A more explicit form of this result is that if perfect state transfer from $u$ to $v$ takes place at time $\tau$, then $H(\tau)$ can be written in block form

$$
\left(\begin{array}{cc}
H_{1} & 0 \\
0 & H_{2}
\end{array}\right)
$$

where $H_{1}$ and $H_{2}$ are unitary and

$$
H_{1}=\gamma\left(\begin{array}{ll}
0 & 1 \\
1 & 0
\end{array}\right)
$$

We also see that $e_{u}+e_{v}$ is an eigenvector for $H(\tau)$ with eigenvalue $\gamma$, while $e_{u}-e_{v}$ is an eigenvector with eigenvalue $-\gamma$. This has consequences for the spectral decomposition of $H(\tau)$, which we will discuss after introducing some associated notation. If $\theta_{1}, \ldots, \theta_{d}$ are the distinct eigenvalues of $A$, then $E_{r}$ is the matrix that represents orthogonal projection onto the eigenspace with eigenvalue $\theta_{r}$. Hence the matrices $E_{1}, \ldots, E_{d}$ are idempotent and symmetric and for any function $f(x)$ defined on the eigenvalues of $A$, we have

$$
f(A)=\sum_{r=1}^{d} f\left(\theta_{r}\right) E_{r} .
$$

It follows that

$$
H(t)=\sum_{r} \exp \left(i \theta_{r} t\right) E_{r}
$$

when $t=\tau$ and we have perfect state transfer from $u$ to $v$ at time $\tau$. We can rewrite this in the form

$$
H(\tau)=\gamma F_{+}-\gamma F_{-}+\sum \exp \left(i \theta_{r} t\right) E_{r}
$$

where the sum is over the integers $r$ such that $\theta_{r}$ is not congruent modulo $2 \pi$ to $\pm \gamma$. The matrices $F_{+}$and $F_{-}$are non-empty sums of the principal idempotents and $F_{+} F_{-}=0$. 


\section{The Ratio Condition}

If $u$ is a vertex in $X$, we define its eigenvalue support to be the set of eigenvalues $\theta_{r}$ of $X$ such that

$$
E_{r} e_{u} \neq 0
$$

Note that

$$
\left\|E_{r} e_{u}\right\|^{2}=e_{u}^{T} E_{r}^{T} E_{r} e_{u}=e_{u}^{T} E_{r}^{2} e_{u}=e_{u}^{T} E_{r} e_{u}
$$

and therefore $\theta_{r}$ lies in the eigenvalue support of $u$ if and only if $\left(E_{r}\right)_{u, u} \neq 0$. The ratio condition is a necessary condition for a graph to be periodic at a vertex. The version we offer here is stated as Theorem 2.2 in [2]; it is an extension of result from Saxena, Severini and Shparlinski [4], which in turn extends an idea used in Christandl et al [1].

3.1 Theorem. Let $X$ be a graph and let $u$ be a vertex in $X$ at which $X$ is periodic. If $\theta_{k}, \theta_{\ell}, \theta_{r}, \theta_{s}$ are eigenvalues in the support of $e_{u}$ and $\theta_{r} \neq \theta_{s}$, then

$$
\frac{\theta_{k}-\theta_{\ell}}{\theta_{r}-\theta_{s}} \in \mathbb{Q} \text {. }
$$

\section{Orbits and Equitable Partitions}

If $u \in V(X)$, then $\operatorname{Aut}(X)_{v}$ denotes the group of automorphisms of $X$ that fix $v$. Our next result says that if perfect state transfer from $u$ to $v$ occurs, then any automorphism of $X$ that fixes $u$ must fix $v$ (and vice versa).

4.1 Lemma. If $X$ admits perfect state transfer from $u$ to $v$, then $\operatorname{Aut}(X)_{u}=$ $\operatorname{Aut}(X)_{v}$.

Proof. We identify the automorphisms of $X$ with the permutation matrices that commute with $A$. Since $H(t)$ is a polynomial in $A$, any permutation matrix from $\operatorname{Aut}(X)$ commutes with $H(\tau)$. If the automorphism associated with $P$ fixes $u$, the $P e_{u}=e_{u}$. If perfect state transfer takes place at time $\tau$ and $H=H(\tau)$, then there is a complex scalar $\gamma$ such that $|\gamma|=1$ and $H e_{u}=\gamma e_{v}$. So

$$
\gamma P e_{v}=P H e_{u}=H P e_{u}=H e_{u}=\gamma e_{v}
$$

and thus $v$ is fixed. As our argument is symmetric in $u$ and $v$, the lemma follows. 
By way of example, suppose $X$ is a Cayley graph for an abelian group $G$. The map that sends $g$ in $G$ to $g^{-1}$ is an automorphism of $G$ and also an automorphism of $X$. The fixed points of this automorphism are the elements of $G$ with order one or two. So if perfect state transfer for 1 to a second vertex $a$ occurs, then $a$ has order two. Consequently perfect state transfer cannot occur on a Cayley graph for an abelian group of odd order. (Another proof of this is presented as Corollary 4.2 in [2].)

A partition $\pi$ of the vertices of a graph $X$ is equitable if, for each ordered pair of cells $C_{i}$ and $C_{j}$ from $\pi$, all vertices in $C_{i}$ have the same number of neighbors in $C_{j}$. (So the subgraph of $X$ induced by a cell is a regular graph, and the subgraph formed by the vertices of two cells and the edges which join them is bipartite and semiregular.) We note that the orbits of any group of automorphisms of $X$ form an equitable partition. The discrete partition, with each cell a singleton, is always equitable; the trivial partition with exactly one cell is equitable if an only if $X$ is regular. For the basics concerning equitable partitions see for example [3, Sction 9.3]. The join of two equitable partitions is equitable, and consequently given any partition of $X$, there is a unique coarsest refinement of it - the join of all equitable partitions which refine it.

If $u \in V(G)$, we use $\Delta_{u}$ to denote the coarsest equitable refinement of the partition

$$
\{\{u\}, V(X) \backslash\{u\}\}
$$

The orbits of the group of automorphisms of $X$ that fix $u$ will be a refinement of $\Delta_{u}$.

If $\pi$ is a partition of $V(X)$, its characteristic matrix is the 01-matrix whose columns are the characteristic vectors of the cells of $\pi$, viewed as subsets of $V(X)$. If we scale the columns of the characteristic matrix so they are unit vectors, we obtain the normalized characteristic matrix of $\pi$. If $P$ and $Q$ are respectively the characteristic and normalized characteristic matrix of $\pi$, then $P$ and $Q$ have the same column space and $Q^{T} Q=I$. The matrix $Q Q^{T}$ is block diagonal, and its diagonal blocks are all of the form

$$
\frac{1}{r} J_{r}
$$

where $J_{r}$ is the all-ones matrix of order $r \times r$, and the size of the $i$-th block is the size of the $i$-th cell of $\pi$. The vertex $u$ forms a singleton cell of $\pi$ if and only if $Q e_{u}=e_{u}$.

We use $|\pi|$ to denote the number of cells of $\pi$. 
4.2 Lemma. Suppose $\pi$ is a partition of the vertices of the graph $X$, and that $Q$ is its normalized characteristic matrix. Then the following are equivalent:

(a) $\pi$ is equitable.

(b) The column space of $Q$ is A-invariant.

(c) There is a matrix $B$ of order $|\pi| \times|\pi|$ such that $A Q=Q B$.

(d) $A$ and $Q Q^{T}$ commute.

If there is perfect state transfer form $u$ to $v$, then it follows from Lemma 4.1 that the orbit partitions of $\operatorname{Aut}(X)_{u}$ and $\operatorname{Aut}(X)_{v}$ are equal. Since equitable partitions are an analog of orbit partitions, it is not entirely unreasonable to view the following result as an extension of this fact.

4.3 Theorem. Let $u$ and $v$ be vertices in $X$. If there is perfect state transfer from $u$ to $v$, then $\Delta_{u}=\Delta_{v}$.

Proof. Let $Q$ be the normalized characteristic matrix of the partition $\Delta_{u}$ and assume $H(t)=\exp (i A t)$. Then $H(t)$ is a polynomial and so $Q H(t)=H(t) Q$. If we have perfect state transfer from $u$ to $v$ at time $\tau$, then

$$
H(\tau) e_{u}=\gamma e_{v}
$$

and accordingly

$$
\gamma Q e_{v}=Q H(\tau) e_{u}=H(\tau) Q e_{u}=H(\tau) e_{u}=\gamma e_{v} .
$$

So $Q e_{v}=e_{v}$ and this implies that $\{v\}$ is a cell of $\Delta_{u}$.

By way of example, consider a distance-regular graph $X$ with diameter $d$. If $u \in V(X)$, then $\Delta_{u}$ is the distance partition with respect to $u$ with exactly $d+1$ cells, where the $i$-th cell is the set of vertices at distance $i$ from $u$. We conclude that if perfect state transfer occurs on $X$, then for each vertex $u$ there is exactly one vertex vertex at distance $d$ from it. In particular perfect state transfer does not occur on strongly regular graphs. (This also follows from some observations in [2, Section 4].) 


\section{Cospectral Vertices}

Vertices $u$ and $v$ in the graph $X$ are cospectral if

$$
\phi(X \backslash u, t)=\phi(X \backslash v, t) .
$$

Of course two vertices that lie in the same orbit of $\operatorname{Aut}(X)$ are cospectral, but there are many examples of cospectral pairs of vertices where this does not hold. A graph is walk regular if any two of its vertices are cospectral. Any strongly regular graph is walk regular.

5.1 Lemma. If $X$ admits perfect state transfer from $u$ to $v$, then $E_{r} u=$ $\pm E_{r} v$ for all $r$, and $u$ and $v$ are cospectral.

Proof. By Cramer's rule,

$$
\left((t I-A)^{-1}\right)_{u, u}=\frac{\phi(X \backslash u, t)}{\phi(X, t)}
$$

and so using the spectral decomposition we find that

$$
\frac{\phi(X \backslash u, t)}{\phi(X, t)}=\sum_{r}\left(t-\theta_{r}\right)^{-1}\left(E_{r}\right)_{u, u},
$$

where

$$
\left(E_{r}\right)_{u, u}=\left\|E_{r} e_{u}\right\|^{2} .
$$

Assume we have perfect state transfer from $u$ to $v$ at time $\tau$ and set $H=$ $H(\tau)$. Then there is a complex number $\gamma$ such that $|\gamma|=1$ and $H e_{u}=\gamma e_{v}$. As $H E_{r}=\exp \left(i \theta_{r} \tau\right) E_{r}$ we have

$$
\exp \left(i \theta_{r} \tau\right) E_{r} e_{u}=\gamma E_{r} e_{v}
$$

and since $E_{r} e_{u}$ and $E_{r} e_{v}$ are real our first claim follows. This implies that

$$
e_{u}^{T} E_{r} e_{u}=\left\|E_{r} e_{u}\right\|^{2}=\left\|E_{r} e_{v}\right\|^{2}=e_{v}^{T} E_{r} e_{v}
$$

and therefore $u$ and $v$ are cospectral. 


\section{Integrality, Nearly}

From [2] we know that if a graph is periodic, then the squares of its eigenvalues are integers and, if the graph is not bipartite, the eigenvalues themselves are integers. Here we derive a variant of this fact, which will imply that that if perfect state transfer occurs on $X$, then the square of the spectral radius of $X$ is an integer.

At the end of the previous section we saw that if we had perfect state transfer from $u$ to $v$ at time $\tau$, then

$$
H(\tau) E_{r} e_{u}=\gamma E_{r} e_{v}
$$

from which it follows that the eigenvalue supports of $u$ and $v$ are equal.

6.1 Theorem. Suppose $X$ is a connected graph with at least three vertices where perfect state transfer from $u$ to $v$ occurs at time $\tau$. Let $\delta$ be the dimension of the $A$-invariant subspace generated by $e_{u}$. Then $\delta \geq 3$ and either all eigenvalues in the eigenvalue support of $u$ are integers, or they are all of the form $\frac{1}{2}\left(a+b_{i} \sqrt{\Delta}\right)$ where $\Delta$ is a square-free integer and $a$ and $b_{i}$ are integers.

Proof. Let $S$ denote the eigenvalue support of $e_{u}$. From the spectral decomposition, the $A$-invariant subspace generated by $e_{u}$ is spanned by the non-zero vectors $E_{r} e_{u}$ such that $\theta_{r} \in S$. Since $E_{r} E_{s}=0$ when $r \neq s$ these vectors are pairwise orthogonal and so form a basis for our $A$-invariant subspace. We conclude that $\delta=|S|$.

Since we have transfer from $u$ to $v$ at time $\tau$, there is a complex number $\gamma$ such that

$$
H(\tau) e_{u}=\gamma e_{v}
$$

from which we see that that the $A$-invariant subspace generated by $e_{u}$ contains $e_{v}$. Hence $\delta \geq 2$, but if equality holds then the span of $e_{u}$ and $e_{v}$ is $A$-invariant and, since $X$ is connected it follows that $|V(X)|=2$.

If $\theta_{r}$ and $\theta_{s}$ are algebraic conjugates, then $E_{r}$ and $E_{s}$ are algebraic conjugates and so $E_{r} e_{u}=0$ if and only if $E_{s} e_{u}=0$. Hence $S$ contains all algebraic conjugates of each of its elements. If two eigenvalues in $S$ are integers, say $\theta_{0}$ and $\theta_{1}$ then since the ratio conditions asserts that

$$
\frac{\theta_{r}-\theta_{0}}{\theta_{1}-\theta_{0}} \in \mathbb{Q},
$$


we conclude that all elements of $S$ are integers.

If all eigenvalues in $S$ are integers, we're done, so suppose that $\theta_{0}$ and $\theta_{1}$ are two distinct irrational eigenvalues in $S$. We show that $\left(\theta_{1}-\theta_{0}\right)^{2}$ is an integer. By the ratio condition, if $\theta_{r}, \theta_{s} \in S$ there is a rational number $a_{r, s}$ such that

$$
\theta_{r}-\theta_{s}=a_{r, s}\left(\theta_{1}-\theta_{0}\right)
$$

and therefore

$$
\prod_{r \neq s}\left(\theta_{r}-\theta_{s}\right)=\left(\theta_{1}-\theta_{0}\right)^{\delta^{2}-\delta} \prod_{i \neq j} a_{r, s} .
$$

The product on the left is an integer and the product of the $a_{r, s}$ 's is rational, and hence

$$
\left(\theta_{1}-\theta_{0}\right)^{\delta^{2}-\delta} \in \mathbb{Q}
$$

Since $\theta_{1}-\theta_{0}$ is an algebraic integer, this implies that

$$
\left(\theta_{1}-\theta_{0}\right)^{\delta^{2}-\delta} \in \mathbb{Z}
$$

Suppose $m$ is the least positive integer such that $\left(\theta_{1}-\theta_{0}\right)^{m}$ is an integer. Then there are $m$ distinct conjugates of $\theta_{1}-\theta_{0}$ of the form

$$
\beta e^{2 \pi i k / m} \quad(k=0, \ldots, m-1)
$$

where $\beta$ is the positive real $m$-th root of an integer, and since the eigenvalues of $X$ are real we conclude that $m \leq 2$. Since $\theta_{1}-\theta_{0}$ is not rational, it must therefore be the square root of an integer. Assume $\Delta=\left(\theta_{1}-\theta_{0}\right)^{2}$.

Let $\mathbb{F}$ be the extension of $\mathbb{Q}$ generated by $S$ and let $G$ be the Galois group of this extension. We aim to show that $|\mathbb{F}: \mathbb{Q}|=2$. Let $\sigma$ be a non-identity element of $G$ and let $\theta$ be an irrational element in $S$. Then

$$
\frac{\theta^{\sigma^{2}}-\theta^{\sigma}}{\theta^{\sigma}-\theta}=a \in \mathbb{Q}
$$

and consequently

$$
\frac{\theta^{\sigma^{k+1}}-\theta^{\sigma^{k}}}{\theta^{\sigma^{k}}-\theta^{\sigma^{k-1}}}=a^{\sigma^{k-1}}=a .
$$

The product of all the possible fractions on the left here is 1 , so if $m$ is the order of $\sigma$, then $a^{m}=1$. Since $a$ is real, this implies that $a= \pm 1$.

If $a=1$, then

$$
\theta^{\sigma^{k+1}}-\theta^{\sigma^{k}}=\theta^{\sigma^{k}}-\theta^{\sigma^{k-1}}
$$


from which it follows that for all $k$

$$
\theta^{\sigma^{k+1}}-\theta=k\left(\theta^{\sigma}-\theta\right)
$$

Setting $k$ equal to the order of $\sigma$ yields a contradiction, and thus $a=-1$. Now we have

$$
\theta^{\sigma^{2}}-\theta^{\sigma}=-\theta^{\sigma}+\theta
$$

and accordingly $\sigma^{2}$ fixes $\theta$. Therefore all elements of the Galois group $G$ have order dividing two.

Now let $\varphi$ be an element of $S$. Since

$$
\frac{\varphi-\theta}{\theta^{\sigma}-\theta} \in \mathbb{Q}
$$

we have

$$
\varphi=a \theta^{\sigma}+(1-a) \theta
$$

for some rational $a$. If $\rho \in G$, then there is a rational $b$ such that

$$
\begin{aligned}
\theta^{\rho} & =b \theta^{\sigma}+(1-b) \theta \\
\theta^{\sigma \rho} & =(1-b) \theta^{\sigma}+b \theta .
\end{aligned}
$$

As $\rho^{2}=1$ and

$$
\left(\begin{array}{cc}
b & 1-b \\
1-b & b
\end{array}\right)^{2}=\left(\begin{array}{cc}
b^{2}+(1-b)^{2} & 2 b(1-b) \\
2 b(1-b) & b^{2}+(1-b)^{2}
\end{array}\right)
$$

we have $b-b^{2}=0$. If $b=0$ then $\rho=1$, if $b=1$ then $\rho=\sigma$. We conclude that $|G|=2$ and $|\mathbb{F}: \mathbb{Q}|=2$.

As $|G|=2$, the field $\mathbb{Q}\left(\theta_{0}\right)$ is a quadratic extension of $\mathbb{Q}$ and consequently $\mathbb{Q}\left(\theta_{0}\right)=\mathbb{Q}(\sqrt{\Delta})$. Because $X$ is connected its spectral radius is simple and the corresponding eigenvector is positive. Therefore the spectral radius must lie in the eigenvalue support $S$ of $e_{u}$. Assume $\theta_{0}$ is the spectral radius and suppose it is an integer. Then if $\theta_{1}$ is an irrational eigenvalue in $S$, we may assume that

$$
\theta_{1}=\theta_{0}-\sqrt{\Delta}
$$

(where $\Delta \in \mathbb{Z}$ ) but then the conjugate $\theta_{0}+\sqrt{\Delta}$ is an eigenvalue of $X$ greater than $\theta_{0}$. Therefore if $\theta_{0}$ is an integer, all elements of $S$ are integers. 
Assume $\theta_{0}$ is irrational, and let $\theta_{0}, \ldots, \theta_{m}$ be the irrational eigenvalues in $S$. Then $\theta_{0}-\theta_{1}=\sqrt{\Delta}$ for some integer $\Delta$ and for $i=0, \ldots, m$ there are rationals $a_{i}$ and $b_{i}$ such that

$$
\theta_{i}=a_{i}+b_{i} \sqrt{\Delta}
$$

(Since $\theta_{i}$ is an algebraic integer, both $2 a_{i}$ and $2 b_{i}$ must be integers.) The conjugate $\bar{\theta}_{i}$ of $\theta_{i}$ is $a_{i}-b_{i} \sqrt{\Delta}$ and, by the ratio condition,

$$
\frac{\theta_{i}-\theta_{0}}{\bar{\theta}_{0}-\theta_{0}}=\frac{a_{i}-a_{0}+\left(b_{i}-b_{0}\right) \sqrt{\Delta}}{2 b_{0} \sqrt{\Delta}} \in \mathbb{Q},
$$

whence $a_{i}=a_{0}$ for $i=0, \ldots, m$.

If there is an integer eigenvalue $\psi$ in $S$, then

$$
\frac{\psi-a_{0}-b_{i} \sqrt{\Delta}}{\psi-a_{0}+b_{i} \sqrt{\Delta}} \in \mathbb{Q} .
$$

Since

$$
\frac{a+b \sqrt{\Delta}}{c+d \sqrt{\Delta}} \in \mathbb{Q} \Longleftrightarrow \operatorname{det}\left(\begin{array}{ll}
a & b \\
c & d
\end{array}\right)=0,
$$

it follows that $\psi=a_{0}$.

Suppose $\delta=3$. Then the subspace $U$ generated by $e_{u}$ contains $e_{v}, A e_{u}$ and $A e_{v}$. If $A e_{u}=e_{v}$, then $\delta=2$, and so $U$ is spanned by $e_{u}, e_{v}$ and either one of $A e_{u}$ and $A e_{v}$. We find that $X$ is the join of $u$ and $v$ with a regular graph.

6.2 Corollary. There are only finitely many connected graphs with maximum valency at most $k$ where perfect state transfer occurs.

Proof. Suppose $X$ is a connected graph where perfect state transfer from $u$ to $v$ occurs at time $\tau$ and let $S$ be the eigenvalue support of $u$. If the eigenvalues in $S$ are integers then $|S| \leq 2 k+1$, and if they are not integers then $|S|<(2 k+1) \sqrt{2}$. So the dimension of the $A$-invariant subspace of $\mathbb{R}^{V(X)}$ generated by $e_{u}$ is at most $\lceil(2 k+1) \sqrt{2}\rceil$, and this is also a bound on the maximum distance from $u$ of a vertex in $X$. If $s \geq 1$, the number of vertices at distance $s$ from $u$ is at most $k(k-1)^{s-1}$, and the result follows. 


\section{Controllable Vertices}

Let $A$ be the adjacency matrix of the graph $X$ on $n$ vertices and suppose $S$ is a subset of $V(X)$ with characteristic vector $e_{S}$. The walk matrix of $S$ is the matrix with columns

$$
e_{S}, A e_{S}, \ldots, A^{n-1} e_{S}
$$

We say that the pair $(X, S)$ is controllable if this walk matrix is invertible. If $u \in V(X)$ then the walk matrix of $u$ is just the walk matrix relative to the subset $\{u\}$ of $V(X)$. From our discussion at the start of the proof of Theorem 6.1, we have that the rank of the walk matrix relative to $u$ is equal to the size of the eigenvalue support of $u$. Thus if $(X, u)$ is controllable, the eigenvalues of $A$ must be distinct.

We say that vertices $u$ and $v$ of $X$ are cospectral if the vertex-deleted subgraphs

$$
X \backslash u, \quad X \backslash v
$$

are cospectral. We use $\phi(X, x)$ to denote the characteristic polynomial of $A(X)$.

7.1 Lemma. Let $u$ and $v$ be vertices of $X$ with respective walk matrices $W_{u}$ and $W_{v}$. Then $u$ and $v$ are cospectral if and only if $W_{u}^{T} W_{u}=W_{v}^{T} W_{v}$.

Proof. By Cramer's rule

$$
\left((x I-A)^{-1}\right)_{u, u}=\frac{\phi(X \backslash u, x)}{\phi(X, x)}
$$

Now

$$
\left((x I-A)^{-1}\right)_{u, u}=x^{-1} \sum_{k \geq 0}\left(A^{k}\right)_{u, u} x^{-k}
$$

and therefore vertices $u$ and $v$ are cospectral if and only if

$$
\left(A^{k}\right)_{u, u}=\left(A^{k}\right)_{v, v}
$$

for all non-negative integers $k$. If $n=|V(X)|$, then equality holds for all non-negative integers $k$ if and only if it holds for $k=0,1, \ldots, n-1$. To complete the proof observe that if $W_{u}$ is the walk matrix for the vertex $u$, then

$$
\left(W_{u}^{T} W_{u}\right)_{r, s}=e_{u}^{T} A^{r+s-2} e_{u} .
$$

One consequence of this is that if $u$ and $v$ are cospectral and $(X, u)$ is controllable, then so is $(X, v)$. We also have: 
7.2 Corollary. If $u$ is a vertex in $X$ with walk matrix $W_{u}$, then $\operatorname{rk}\left(W_{u}\right)$ is equal to the number of poles of the rational function $\phi(X \backslash u, x) / \phi(X, x)$. Hence $(X, u)$ is controllable if and only if $\phi(X \backslash u, x)$ and $\phi(X, x)$ are coprime.

Proof. By the spectral decomposition,

$$
\left((x I-A)^{-1}\right)_{u, u}=\sum_{r} \frac{1}{x-\theta_{r}}\left(E_{r}\right)_{u, u} .
$$

We will prove that a controllable vertex cannot be involved in perfect state transfer. For this we need the following:

7.3 Lemma. Let $X$ be a graph with $n=|V(X)|$. If $\sigma$ is the minimum distance between two eigenvalues of $X$, then

$$
\sigma^{2}<\frac{12}{n+1}
$$

Proof. Assume that the eigenvalues of $X$ in non-increasing order are $\theta_{1}, \ldots, \theta_{n}$. If we have

$$
M:=A \otimes I-I \otimes A
$$

then the eigenvalues of $M$ are the numbers

$$
\theta_{i}-\theta_{j}, \quad 1 \leq i, j \leq n .
$$

Now

$$
M^{2}=A^{2} \otimes I+I \otimes A^{2}-2 A \otimes A
$$

and consequently, if $e:=|E(X)|$ then

$$
\sum_{i, j=1}^{n}\left(\theta_{i}-\theta_{j}\right)^{2}=\operatorname{tr}\left(M^{2}\right)=2 n \operatorname{tr}\left(A^{2}\right)=4 n e .
$$

Since

$$
\theta_{i}-\theta_{j} \geq(i-j) \sigma
$$

we have

$$
\sum_{i, j=1}^{n}\left(\theta_{i}-\theta_{j}\right)^{2} \geq \sigma^{2} \sum_{i, j=1}^{n}(i-j)^{2}
$$

As

$$
\sum_{i=1}^{n} i^{2}=\frac{n(n+1)(2 n+1)}{6}
$$


we find that

$$
\sum_{i, j=1}^{n}(i-j)^{2}=2 n \sum_{i=1}^{n} i^{2}-2\left(\sum_{i=1}^{n} i\right)^{2}=\frac{n^{2}\left(n^{2}-1\right)}{6},
$$

and since $e \leq n(n-1)$ this yields

$$
\sigma^{2} \frac{n^{2}\left(n^{2}-1\right)}{6} \leq 4 n e \leq 2 n^{2}(n-1) .
$$

This gives our stated bound but with $\leq$ in place of $<$. To achieve strictness we note that if equality were to hold then $e=n(n-1)$ and $X=K_{n}$. Since $\sigma\left(K_{n}\right)=0$, we are done.

7.4 Theorem. Let $X$ be a connected graph on at least four vertices. If we have perfect state transfer between distinct vertices $u$ and $v$ in $X$, then neither $u$ nor $v$ is controllable.

Proof. Let $n=|V(X)|$. We assume by way of contradiction that $(X, u)$ is controllable. Then the eigenvalue support of $u$ contains all eigenvalues of $X$ and these eigenvalues are distinct. By Theorem 6.1, this means that there are integers $a$ and $\Delta$ and distinct integers $b_{1}, \ldots, b_{n}$ and the eigenvalues of $A$ are the numbers

$$
\frac{1}{2}\left(a+b_{r} \sqrt{\Delta}\right) .
$$

As $\operatorname{tr}(A)=0$ we have

$$
0=\frac{1}{2} \sum_{r=1}^{n}\left(a+b_{i} \sqrt{\Delta}\right)=\frac{n a}{2}+\frac{\sqrt{\Delta}}{2} \sum_{r=1}^{n} b_{r}
$$

If $\sqrt{\Delta}$ is not an integer, this implies that $\sum_{r} b_{r}=0$ and hence that $a=0$. Accordingly the eigenvalues of $A$ are distinct integer multiples of the irrational number $\sqrt{\Delta}$ that sum to zero. If $\sqrt{\Delta}$ is an integer then the eigenvalues of $A$ are distinct integers that sum to zero. In either case $\sigma(X) \geq 1$.

By Lemma 7.3 we can assume that $n=\mid V(X) \leq 10$. This leaves us with six cases. First suppose $\Delta=1$. Assume $n=10$. Then the sum of the squares of the eigenvalues of $X$ is bounded below by the sum of the squares of the integers from -4 to 5 , which is 85 , and hence the average valency of a vertex 
is at least 8.5. This implies that $\theta_{1}=9$, not 5 , consequently the sum of the squares of the eigenvalues is at least

$$
85-25+81=141
$$

and now the average valency is 14.1 , which is impossible. The cases where $n$ is 7,8 or 9 all yield contradictions in the same way. If $\Delta>1$, it is even easier to derive contradictions.

Next, brute force computation (using Sage [5]) shows that the path $P_{4}$ is the only graph on 4,5 or 6 vertices where the minimum separation between consecutive eigenvalues is at least 1 . The positive eigenvalues of $P_{4}$ are

$$
(\sqrt{5} \pm 1) / 2
$$

and their ratio is not rational.

\section{$8 \quad$ Nearly Perfect State Transfer?}

If $u$ and $v$ are controllable and cospectral we cannot get perfect state transfer between them. Our next results shows that if these conditions hold, there is a symmetric orthogonal matrix $Q$ which commutes with $A$ and maps $e_{u}$ to $e_{v}$.

8.1 Lemma. Let $u$ and $v$ be vertices in $X$ with respective walk matrices $W_{u}$ and $W_{v}$. If $u$ and $v$ are controllable and $Q:=W_{v} W_{u}^{-1}$ then $Q$ is a polynomial in $A$. Further $Q$ is orthogonal if and only if $u$ and $v$ are cospectral.

Proof. Let $C_{\phi}$ denote the companion matrix of the characteristic polynomial of $A$. Then

$$
A W_{u}=W_{u} C_{\phi}
$$

for any vertex $u$ in $X$. Hence if $u$ and $v$ are controllable,

$$
W_{u}^{-1} A W_{u}=W_{v}^{-1} A W_{v}
$$

and from this we get that

$$
A W_{v} W_{u}^{-1}=W_{v} W_{u}^{-1} A .
$$


Since $X$ has a controllable vertex its eigenvalues are all simple, and so any matrix that commutes with $A$ is a polynomial in $A$. This proves the first claim.

From Lemma 7.1, the vertices $u$ and $v$ are cospectral if and only if $W_{u}^{T} W_{u}=W_{v}^{T} W_{v}$, which is equivalent to

$$
Q^{-T}=W_{v}^{-T} W_{u}^{T}=W_{v} W_{u}^{-1}=Q
$$

This places us in an interesting position. If $u$ and $v$ are cospectral and controllable, there is an orthogonal matrix $Q$ that commutes with $A$ such that $Q e_{u}=e_{v}$ but, by the result of the previous section, $Q$ cannot be equal to a scalar multiple of $H(t)$ for any $t$.

\section{Acknowledgement}

I thanks Dave Morris for carefully reading the first draft of this paper and for providing a number of useful comments.

\section{References}

[1] M. Christandl, N. Datta, T. Dorlas, A. Ekert, A. Kay, And A. LANDAhl, Perfect transfer of arbitrary states in quantum spin networks, Phys Rev A, 71 (2005), p. 032312.

[2] C. Godsil, Periodic graphs, arXiv, math.CO (2008), 0806.2074v2. 19 pages.

[3] C. Godsil and G. Royle, Algebraic Graph Theory, vol. 207 of Graduate Texts in Mathematics, Springer-Verlag, New York, 2001.

[4] N. Saxena, S. Severini, And I. Shparlinski, Parameters of integral circulant graphs and periodic quantum dynamics, arXiv, quant-ph (2007), quant-ph/0703236v1. 12 pages.

[5] W. Stein et AL., Sage Mathematics Software (Version 4.5.2), The Sage Development Team, 2010. http://www.sagemath.org. 